\title{
Tuning Reactivity of Micellar Nanoreactors by Precise Adjust- ments of the Amphiphiles and Substrates Hydrophobicity
}

\author{
Shahar Tevet ${ }^{\ddagger 1,2}$, Shreyas Shankar Wagle ${ }^{\ddagger 1,2}$, Gadi Slor ${ }^{1,2}$ and Roey J. Amir ${ }^{* 1,2,3,4,5}$ \\ 1 Department of Organic Chemistry, School of Chemistry, Faculty of Exact Sciences, Tel-Aviv University, Tel-Aviv 6997801, Israel \\ 2 Tel-Aviv University Center for Nanoscience and Nanotechnology, Tel-Aviv University, Tel-Aviv 6997801, Israel \\ 3 BLAVATNIK Center for Drug Discovery, Tel-Aviv University, Tel-Aviv 6997801, Israel \\ 4 ADAMA Center for Novel Delivery Systems in Crop Protection, Tel-Aviv University, Tel-Aviv 6997801, Israel \\ 5 The Center for Physics and Chemistry of Living Systems, Tel-Aviv University, Tel-Aviv 6997801, Israel
}

KEYWORDS: Polymeric Micelles, Micellar Nanoreactor, Micellar catalysis, Organometallic reactions, Green Chemistry, Bioorthogonal Chemistry.

\begin{abstract}
Polymeric assemblies, such as micelles, are gaining increasing attention due to their ability to serve as nanoreactors for the execution of organic reactions in aqueous media. The ability to conduct transformations, which have been limited to organic media, in water is essential for the further development of the important fields of green catalysis and biorthogonal chemistry, among other fields. In light of the recent progress in the expanding the scopes of reactions that can be conducted using nanoreactors, we aimed to gain deeper understanding of the roles of the hydrophobicity of both the core of micellar nanoreactors and the substrates on the reaction rates in water. Towards this goal we designed a set of metal-loaded micelles, composed of PEG-dendron amphiphiles and studied their ability to serve as nanoreactors for a palladium mediated depropargylation reaction of four substrates with different $\log P$ values. Using dendrons as the hydrophobic block, allowed us to fine tune the lipophilicity of the dendritic end-groups and study how precise structural changes in the hydrophobicity of the amphiphiles affect the reaction rates. The kinetic data revealed linear relations between the rate constants and the hydrophobicity of the amphiphiles (estimated by the dendron's cLogP), while exponential dependence was obtained for the lipophilicity of the substrates (estimated by their LogP values). Our results demonstrate the vital contributions of the hydrophobicity of both the substrates and amphiphiles on the lipo-selectivity of nanoreactors, illustrating the potential of tuning hydrophobicity as a tool for optimizing the reactivity and selectivity of nanoreactors.
\end{abstract}

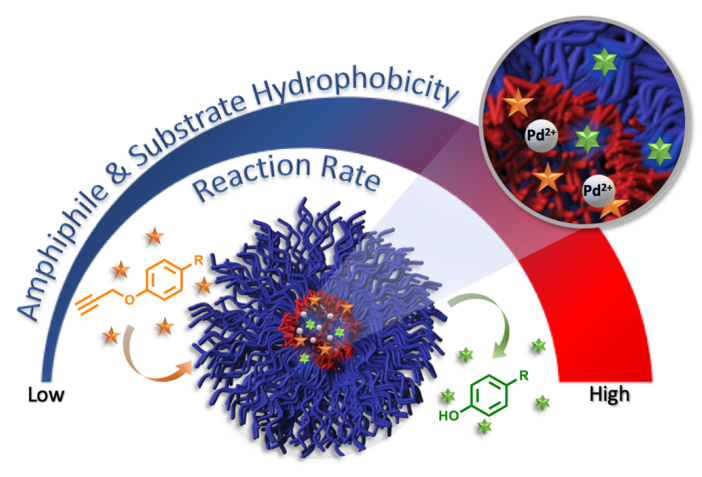




\section{INTRODUCTION}

While an aqueous environment is essential for all living systems, using water as a solvent does not necessarily translate well for conducting organic reactions and in particular organometallic chemistry. The limited applicability of water as reaction media emerges from the fact that the majority of the chemicals used in such reactions are lipophilic, in addition to the risk of poisoning the reactive species/catalysts by water molecules. Nevertheless, the development of methodologies for conducting organic reactions in aqueous media has a significant influence on various fields, from synthetic biology and therapeutic biomaterials to green chemistry. ${ }^{1-9}$

Polymeric micelles can act as nanometer-sized flasks for conducting organic reactions in water. ${ }^{10}$ Micelles can provide solubility and protective environment for the lipophilic reactants, shielding them from the surrounding aqueous environment. ${ }^{11,12}$ In recent years, significant progress in conducting organic transformation and specifically organometallic reactions has been reported by Lipshutz, ${ }^{12-17}$ Meijer, ${ }^{3,18,19}$ Unciti-Broceta ${ }^{5,20-24}$ Zimmerman, $^{25-27}$ and others. ${ }^{28-32}$ The ability to perform organometallic reactions in aqueous media can contribute significantly to increasing the sustainability of organic synthesis by reducing the usage of organic solvents. Furthermore, the development of metal loaded nanoreactors can open new horizons for broadening the scope of bioorthogonal approaches. Furthermore, Despite the great progress in this field, the rational design of micellar systems as nano reaction-vessels is still a huge chemical challenge, mostly due to the lack of broader knowledge on the structure-activity relations of these systems.

To gain better understanding of the function of micellar nanoreactors as reaction media, we aimed to systematically study the influence of the hydrophobicity of both the core of the micelles and the substrate on reaction rates in water. We chose the palladium-mediated O-propargyl cleavage, ${ }^{33-42}$ as a model reaction, due to its potential to serve as bioorthogonal approach for the activation of prodrugs. ${ }^{4,5,7,43}$ Towards this study, we developed metal-embedded micellar system, using high molecular precision linear-dendritic amphiphiles. The usage of dendrons with different alkyl end-groups as the hydrophobic blocks of the amphiphiles was envisioned to grant us maximal molecular control over the lipophilicity of the micellar core when studying the rates of our model reaction. To evaluate the impact of the substrate's hydrophobicity on the reaction rate, we also synthesized low molecular weight propargyl-modified compounds with different degree of lipophilicities and used them in our model depropargylation reaction. This methodology allowed us to estimate the individual impact of the lipophilic microenvironment of the micellar core and the hydrophobicity of the substrates on the reaction rate (Figure 1).

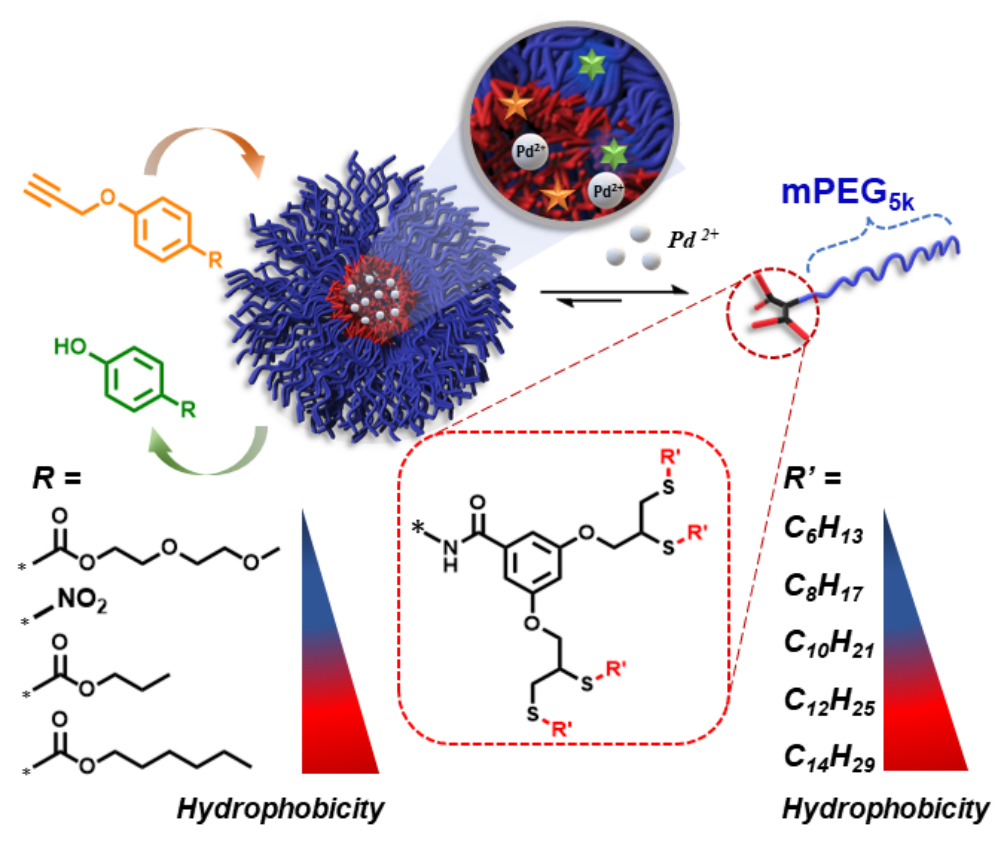

Figure 1. Schematic illustration of palladium loaded micellar nanoreactors based on PEG-dendron amphiphiles for the depropargylation of substrates with increasing degree of lipophilicity. 


\section{RESULTS AND DISCUSSION}

\section{Molecular design and synthesis of amphiphiles}

For the micellar framework, we designed amphiphilic hybrids based on a commercial 5kDa mono-functional polyethylene glycol (mPEG) as a hydrophilic block, and a dendron with four aliphatic end-groups as the hydrophobic block. For the metal catalyst, we chose to work with a simple palladium (II) acetate salt, which has poor aqueous solubility. The hydrophobic micellar core should encapsulate the lipophilic metal salt, affording its solubilization in water, as well as providing the required microenvironment for the organometallic reaction. Our synthetic methodology was aimed to be modular and step-efficient. In addition, it gave us high molecular control over the degree of hydrophobicity by simply tuning the length of the aliphatic end-groups. The amphiphiles were synthesized in only two high yielding steps, starting by conjugating $\mathrm{mPEG}$-amine $\left(\mathrm{mPEG}_{5 \mathrm{k}}-\mathrm{NH}_{2}\right)$ with an activated para-nitrophenol ester of di-propargyl branching unit (PNP-BU) to yield a stable amide bond. Thiol-yne reaction of the di-alkyne functionalized mPEG $\left(\mathrm{mPEG}_{\mathrm{sk}}\right.$-diyne) with five different linear aliphatic thiols with lengths ranging from 6 to 14 carbons, allowed us to produce dendritic structure containing two 1,2 di-mercaptoethers moieties in each dendron (Scheme 1). ${ }^{1} \mathrm{H}-\mathrm{NMR}$, GPC, HPLC and MALDI-TOF MS measurements were used to verify the synthetic conversion and the product's purity and polydispersity, and the experimental results showed excellent correlation with the expected values as can be seen in the supporting information.

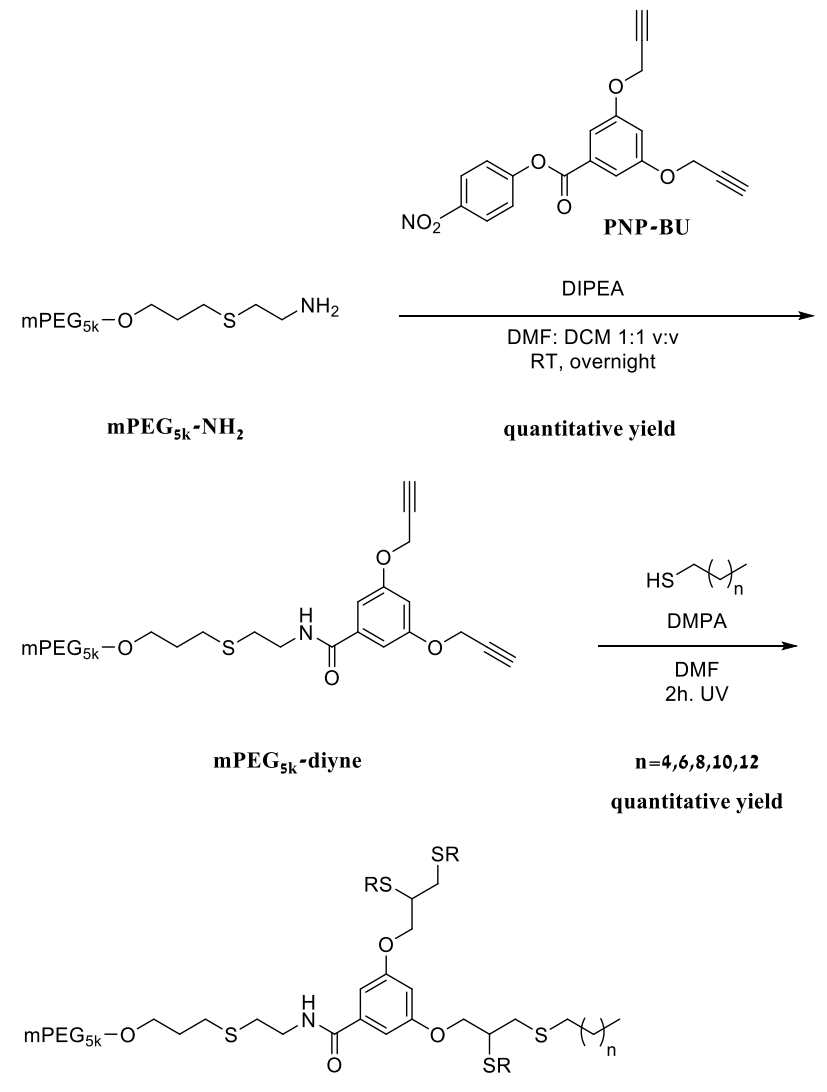

$$
\begin{gathered}
\text { CX amphiphile } \\
\text { mPEG }_{5 \mathrm{k}}-\mathrm{D}-(\mathrm{CX})_{4} \\
\mathrm{X}=6,8,10,12,14
\end{gathered}
$$

Scheme 1. Synthetic route for $\mathrm{mPEG}_{5 \mathrm{k}}-\mathrm{D}-(\mathrm{CX})_{4}$ amphiphilic hybrids. X refers to the number of carbons at the aliphatic chain. 


\section{Self-assembly into micellar nanoreactors}

Once completing the synthesis of the five amphiphiles, we wished to examine their self-assembly into micellar struc-

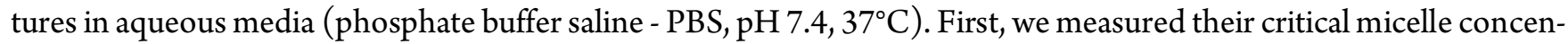
tration (CMC) values using the Nile Red method. ${ }^{44}$ Due to the minor differences of only few carbons between the chemical structures of the amphiphiles, we expected a slight decrease in the CMC value as the length of the aliphatic end-groups increased. As seen in table 1 and Figure S17-S22, the CMC values for $\mathrm{mPEG}_{5 \mathrm{k}}-\mathrm{D}-(\mathrm{CX})_{4}$ amphiphiles ranged between $4 \pm 1 \mu \mathrm{M}$ for the $\mathrm{C} 6$ amphiphile to $3 \pm 1 \mu \mathrm{M}$ for the $\mathrm{C} 14$ amphiphile. The obtained values indeed showed the expected trend, which correlates well with previous reports. ${ }^{45}$

After confirming the self-assembly of nanostructures in aqueous environment, we used dynamic light scattering (DLS) to measure the sizes of the formed structures. We determined the diameters of the assembled structures to be in the range of 14-31 nm (table 1), which indicates that the amphiphiles self-assembled into nano-sized micelles. As expected, the amphiphiles with longer hydrophobic aliphatic end-groups formed larger structures, while the ones with shorter end-groups gave smaller sizes. It is interesting to note that although the change in the length of the end-groups is relatively small, a disproportional increase in diameter was observed, indicating on increased packing and higher aggregation numbers of polymeric amphiphiles with longer aliphatic end-groups. ${ }^{46}$ Transmittance electron microscopy (TEM) was further used to validate the formation of spherical structures with similar diameters (Figure S24).

Since the vicinal ether/thio-ether functional groups present throughout the dendritic architecture can potentially act as chelating sites for the palladium, we wished to assess the effect of the presence of the metal salt on the nanostructures. We combined the amphiphiles and the palladium acetate salt at a 1:2 molar ratio in acetone and mixed them briefly. After evaporation of the organic solvent, the mixture was re-dissolved in PBS and DLS size measurements were conducted. While for the metal-free nanostructures, we could observe a clear trend, where the amphiphiles with longer hydrophobic aliphatic end-groups formed larger structures, metal addition to the micelles changed the tendency drastically. Interestingly, in the presence of palladium salt, the different amphiphiles self-assembled into smaller structures with relatively similar sizes in the range of $12-16 \mathrm{~nm}$ (table 1 ). Whereas the diameter for all amphiphiles was reduced, the most significant change in diameter was measured for the C14 amphiphile - from a $31 \mathrm{~nm}$ without the metal to only $13 \mathrm{~nm}$ after its addition. The results confirm the formation of micelles also in the presence of the metal salt and suggest a change in the packing of the micellar structures making them more compact, which might indicate the formation of palladium complex within the micellar core. TEM images provided further validation of the smaller sizes and spherical shape of the metal loaded micelles (Figure S24).

To further evaluate whether there is a formation of palladium complexes within the dendritic branches in the micelles, we performed a complexation experiment using a combination of $\mathrm{C} 12$ amphiphile and the palladium acetate salt. We prepared a series of solutions with different molar ratios of metal to amphiphile, ranging between $0 \%$ to $200 \%$, in $\mathrm{CDCl}_{3}$ and monitored the complex formation by ${ }^{1} \mathrm{H}-\mathrm{NMR}$ (Figure S25). The results show that upon increasing percentage of the metal salt, the peaks of the protons near the vicinal ether/thio-ether groups were significantly broadened until entirely disappeared from the spectrum. This peak broadening is indicative of complex formation at or near these sites, causing decreased mobility and hence short relaxations times. It is interesting to notice that besides the mentioned sites in position $\alpha$ and $\beta$ to the thio-ethers, the rest of the carbons of the aliphatic end-groups did not seem to be affected by the complexation.

\section{Studying the effect of hydrophobicity on the rate of the depropargylation reaction}

\section{Tuning the nanoreactor's hydrophobicity}

Once the palladium loaded micelles were characterized, we wished to examine their ability to conduct the depropargylation reaction and study the effect of increasing the hydrophobicity of the amphiphile on the reaction rate. As a model substrate for the depropargylation reaction, we synthesized a PNP-propargyl ether (PNPPE), which upon cleavage of O-propargyl group should transform back to PNP. Importantly, the PNP product has a lower logP value 
in comparison to the PNPPE substrate (Table 2), indicative of its higher solubility in water. This may facilitate the reaction, which is likely to take place inside the hydrophobic microenvironment of the micellar core. The hydrophobic substrate will tend to migrate into the micellar structures due to its low solubility, and once transformed into the more hydrophilic product, it will be able to migrate back to the outer aqueous environment.

Table 1. Amphiphiles and their properties

\begin{tabular}{|l|l|l|l|l|l|l|l|l|l|}
\hline Amphiphile & End-group & $\begin{array}{l}\mathrm{M}_{\mathrm{n}}{ }^{\mathrm{a}} \\
(\mathrm{kDa})\end{array}$ & $\mathrm{D}$ & $\begin{array}{l}\mathrm{M}_{\mathrm{p}}^{\mathrm{b}} \\
(\mathrm{kDa})\end{array}$ & $\begin{array}{l}\mathrm{M}_{\mathrm{n}}{ }^{\mathrm{c}} \\
(\mathrm{kDa})\end{array}$ & $\begin{array}{l}\mathrm{CMC}^{\mathrm{d}} \\
(\mu \mathrm{M})\end{array}$ & $\begin{array}{l}\mathrm{D}_{\mathrm{H}}{ }^{\mathrm{e}} \\
(\mathrm{nm})\end{array}$ & $\begin{array}{l}\mathrm{D}_{\mathrm{H}}{ }^{\mathrm{f}} \\
(\mathrm{nm})\end{array}$ & $\mathrm{cLogP}^{\mathrm{g}}$ \\
\hline C6 & Hexyl & 5.0 & 1.08 & 5.8 & 5.8 & $4 \pm 1$ & $14 \pm 1$ & $12 \pm 3$ & 13.2 \\
\hline C8 & Octyl & 5.6 & 1.04 & 6.0 & 5.9 & $4 \pm 1$ & $21 \pm 3$ & $13 \pm 3$ & 17.4 \\
\hline C10 & Decyl & 5.9 & 1.05 & 6.1 & 6.0 & $3 \pm 1$ & $23 \pm 4$ & $15 \pm 2$ & 21.7 \\
\hline C12 & Dodecyl & 6.1 & 1.05 & 6.2 & 6.2 & $3 \pm 1$ & $23 \pm 5$ & $16 \pm 4$ & 25.9 \\
\hline C14 & Tetradecyl & 6.2 & 1.05 & 6.4 & 6.3 & $3 \pm 1$ & $31 \pm 4$ & $13 \pm 8$ & 30.1 \\
\hline
\end{tabular}

Table 1. (a) Measured by GPC using PEG commercial standards. (b) Measured by MALDI-TOF MS. (c) Calculated based on mPEG5kDa and the expected exact mass of the dendrons. (d) Determined using Nile red method. (e) Hydrodynamic diameter measured by DLS of micelles formed from amphiphiles only. (f) Hydrodynamic diameter measured by DLS of micelles with encapsulated $\mathrm{Pd}(\mathrm{OAc})_{2}$ salt. $(\mathrm{g})$ Calculated for only the dendritic group of the amphiphile via ChemDraw Version 18.2.

To prepare the metal loaded micelles, the palladium salt and the different amphiphiles were dissolved separately in acetone, mixed and stirred briefly, followed by evaporation of the organic solvent and rehydration in PBS as described before. Finally, the PNPPE substrate was added and the reaction was monitored by HPLC. A sample of amphiphile and substrate in the absence of palladium was used as a control for monitoring the stability of the substrate solution over time and to ensure its hydrolysis cannot be catalyzed by the micellar system alone. A control of substrate and metal in the absence of the micellar structures could not be measured since both compounds have poor water solubility.

To evaluate the propargyl cleavage rate of the substrate, we measured the area under the curve (AUC) of the substrate's peak at each chromatogram (Figure 2A) and plotted the decrease in concentration (in \%) as a function of time (Figure 2B). To evaluate the kinetics of the reaction, a natural log of the normalized experimental data was plotted against time (figure $2 \mathrm{C}$ ), providing a linear equation correlating with first-order reaction $\ln [A]=-k t+\ln [A]_{0}$. The rate constant $(\mathrm{k})$ values were calculated based on the above first-order equation and the theoretical half-life $\left(\mathrm{t}_{1 / 2}\right)$ values were calculated from $t_{1 / 2}=\ln (2) / k$. The $k$ and $t_{1 / 2}$ values are presented alongside the experimental value of $t_{1 / 2}$ in table 2 . These experiments indicate that the reaction occurs faster as the aliphatic end-groups are longer, with $\mathrm{k}$ values being almost double when changing from $\mathrm{C} 6$ to the $\mathrm{C} 14$ amphiphile (table 2). These results highlight the importance of the hydrophobicity of the nanoreactor in the execution of the propargyl cleavage reaction. Furthermore, it demonstrates how small structural changes, of only few carbons in the hydrophobic block of the amphiphiles, can have a remarkable influence on the activity of the nanoreactor.

To compare between the different amphiphiles and gain an indication for the influence of the amphiphiles' hydrophobicity on the reaction rate, the calculated $\mathrm{k}$ values were plotted against the dendrons' cLogP values (figure 2D). Although it is clear that the amphiphile's overall hydrophobicity should be lower due to the hydrophilic PEG block, 
the dendrons' cLogP could give us a quantitative parameter that is a key part of the total hydrophobicity of the amphiphile. The results indicate a linear correlation between the cLogP of the dendrons and the reaction rates, emphasizing the importance of the hydrophobicity of the nanoreactors in the execution of the reaction.

A

C
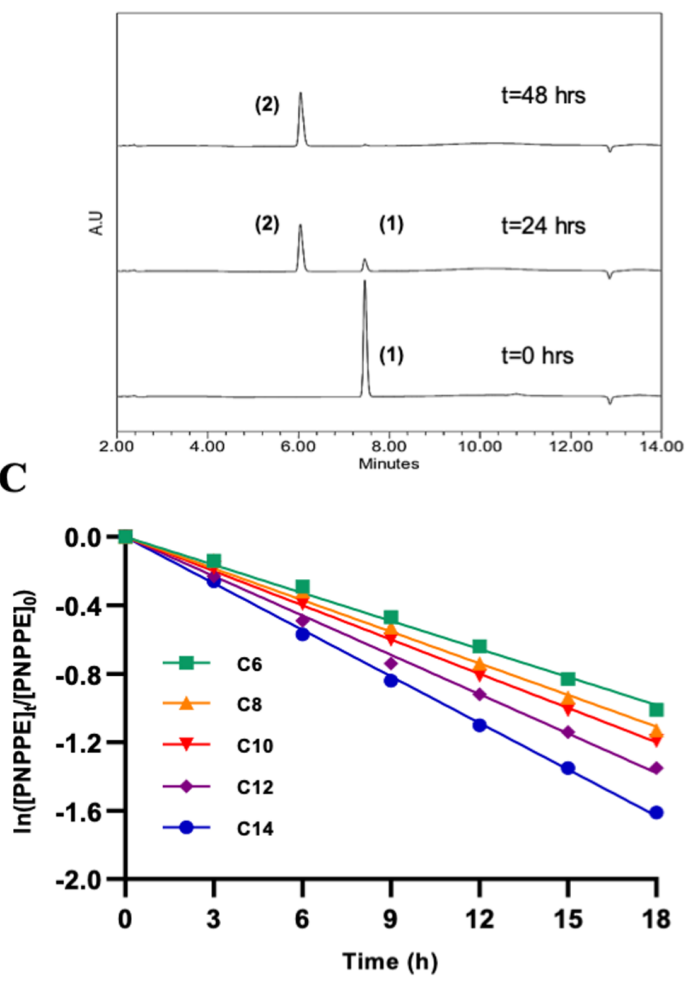

B

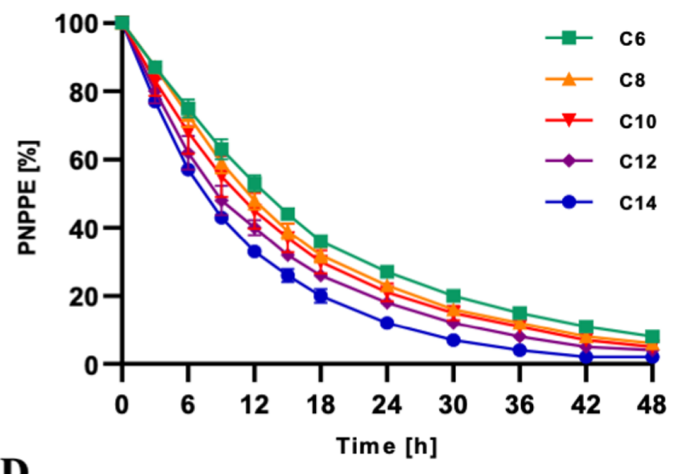

D

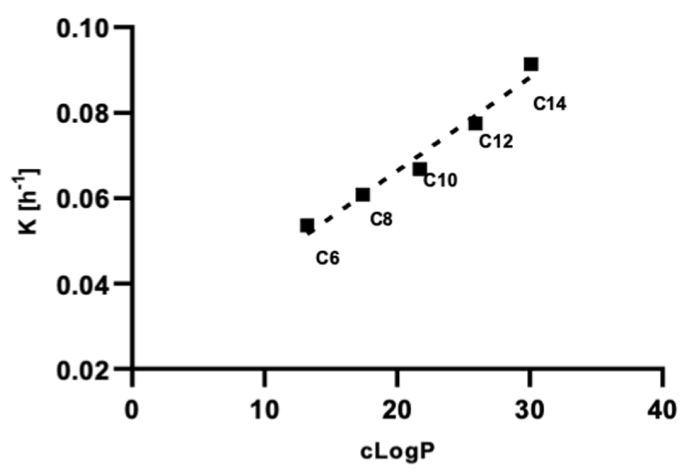

Figure 2. O-Propargyl cleavage kinetics of PNPPE substrate after treatment with metal-loaded micelles composed from amphiphiles with different degrees of hydrophobicity; A) Representative HPLC chromatogram overlay (taken at $307 \mathrm{~nm}$ ), showing the transformation of PNPPE (1) to PNP (2). [Amphiphile] $=42 \mu \mathrm{M} ;\left[\mathrm{Pd}(\mathrm{OAc})_{2}\right]=83 \mu \mathrm{M} ;[\mathrm{PNPPE}]=166 \mu \mathrm{M}$. B) Normalized PNPPE consumption over time. C) Natural log of the normalized experimental kinetic data. D) cLogP values of the amphiphiles' dendrons plotted against the calculated rate constants.

After observing the linear influence of the hydrophobicity of the nanoreactor on the propargyl-cleavage reaction for the PNPPE substrate, we wished to repeat these experiments using a different propargyl-containing substrate. We decided to synthesize 4-(propargyloxy)benzoic acid propyl ester (PropylBPE, Figure 4) in order to examine whether the kinetic trends will be kept or were they specific to the PNPPE substrate.

The PropylBPE substrate was incubated with all five different metal containing micelles, following the same protocol as described above and the reaction was monitored by HPLC. The kinetic results (Figure 3) demonstrated a remarkable similar trend as obtained for the propargyl-cleavage for PNPPE, showing a linear correlation between the calculated $\mathrm{k}$ values and the cLogP of the dendrons. Thus, indicating that the effect of the nanoreactor's hydrophobicity on the reaction rate isn't limited to a specific substrate. Although the linear trend was preserved, we have noticed that the reaction rates were faster for the PropylBPE substrate (table 2). The Propyl-based substrate is more lipophilic and has a higher $\log \mathrm{P}$ value than PNPPE substrate, which indicate its higher tendency to migrate into the hydrophobic core of the micelles, resulting in faster reaction rate. The higher $\mathrm{k}$ values for the PropylBPE in comparison to the PNPPE imply that the hydrophobicity of the substrate has additional effect on the reaction rate in presence of the micellar nanoreactors. 
A
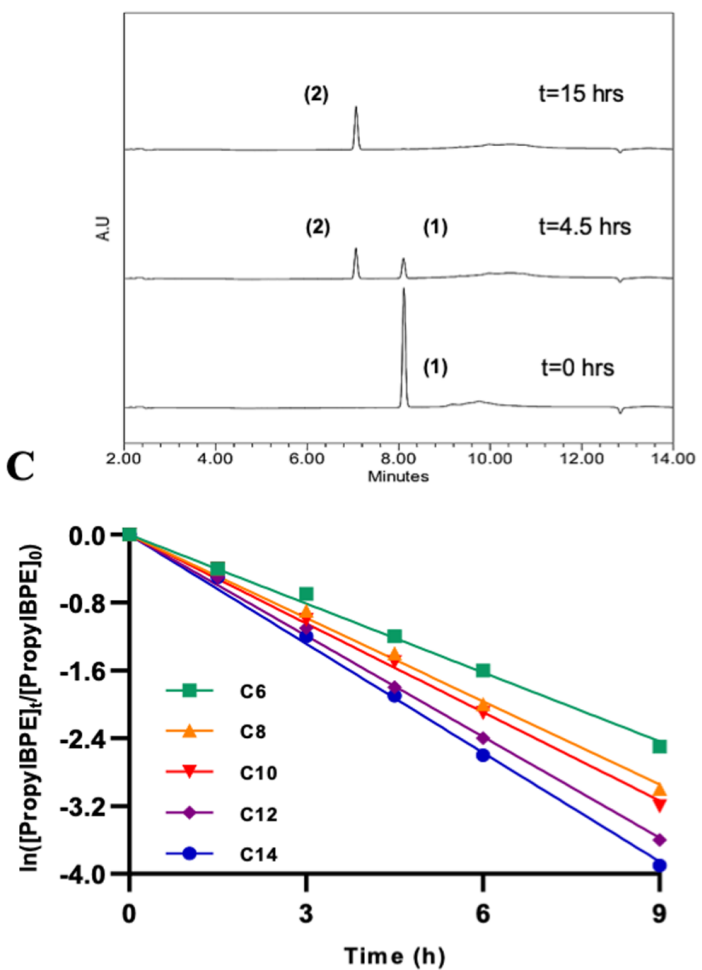

B
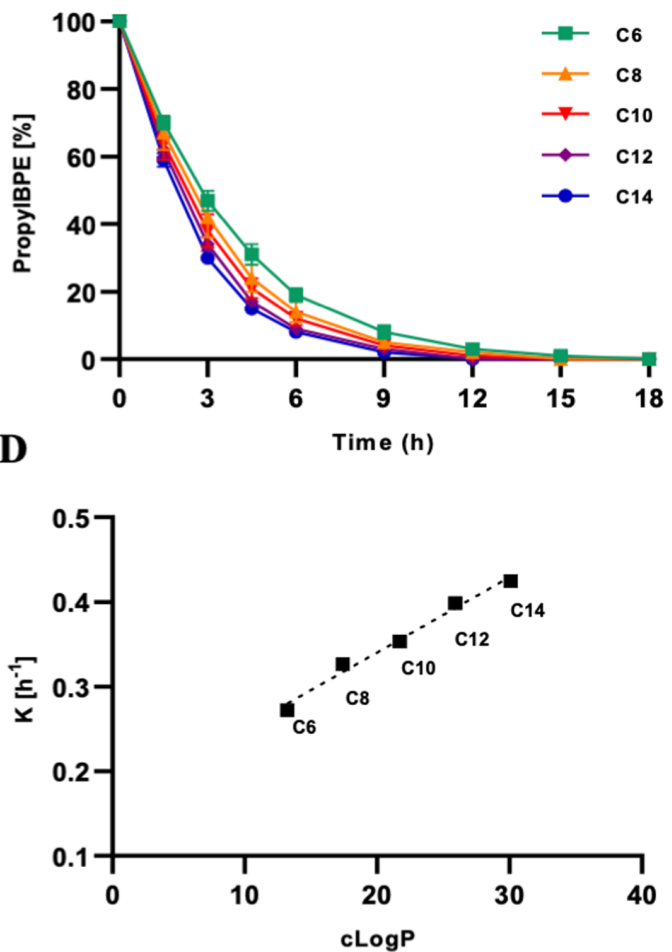

Figure 3. O-Propargyl cleavage profile of Propyl substrate after treatment with metallic-micelles composed from amphiphiles with different degrees of hydrophobicity; A) Representative HPLC chromatogram overlay (taken at $252 \mathrm{~nm}$ ), showing the transformation of Propyl substrate (1) to its' de-propargylated product (2). [Amphiphile] $=42 \mu \mathrm{M}$; $[\mathrm{Pd}(\mathrm{OAc}) 2]=83 \mu \mathrm{M}$; [PropylBPE] = $100 \mu \mathrm{M}$. B ) Normalized Propyl consumption over time. C) Natural log of the normalized experimental kinetic data. D) cLogP values of the amphiphiles' dendrons plotted against their corelated calculated rate constant.

\section{Adjusting the substrates' hydrophobicity}

To evaluate how the hydrophobicity of the substrate will affect the reaction rate, we decided to design two additional derivatives of the Propyl-containing substrate, which would have either higher or lower lipophilicity. The two additional substrates were synthesized by replacing the propyl-ester group with either hexyl or diethylene glycol (DEG) ester, yielding HexylBPE and DEGBPE substrates, respectively (Figure 4).

To examine the effect of the hydrophobicity of the substrate, HexylBPE and DEGBPE were incubated with C6 and $\mathrm{C} 14$ based micellar nanoreactors, following the same protocol as described above. The propargyl cleavage profiles and reaction rates of the different substrates are presented in Figures 5 (A and B) and table 2, alongside with the kinetic data acquired for the Propyl-based substrate. 

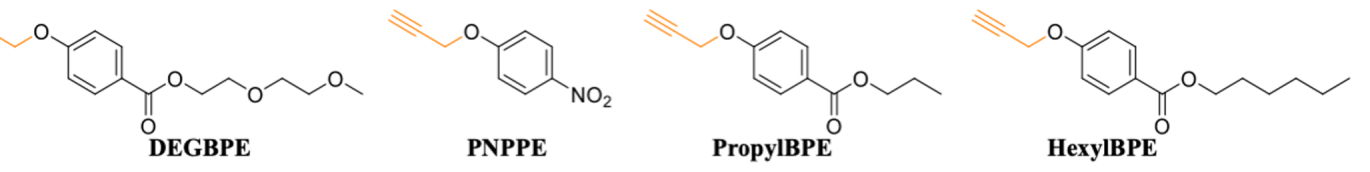

\section{Lipophilicity}

Figure 4. Structures of substrates for the depropargylation reaction.

Table 2. Propargyl containing substrates - properties and kinetic analysis.

\begin{tabular}{|c|c|c|c|c|c|c|c|}
\hline Entry & Substrate & $\begin{array}{l}\text { Substrate } \\
\operatorname{LogP}^{*}\end{array}$ & $\begin{array}{l}\text { Product } \\
\log \mathrm{P}^{*}\end{array}$ & Amphiphile & $\mathrm{k}\left(\mathrm{h}^{-1}\right)$ & $\mathrm{t}_{1 / 2}$ calc. (h) & $t_{1 / 2} \exp .(h)$ \\
\hline 1 & \multirow{6}{*}{ PNPPE } & \multirow{6}{*}{2.1} & \multirow{6}{*}{1.4} & $\begin{array}{l}\text { no am- } \\
\text { phiphile* }\end{array}$ & 0.077 & 9.0 & 7.5 \\
\hline 2 & & & & C6 & 0.054 & 12.9 & 12.7 \\
\hline 3 & & & & C8 & 0.061 & 11.4 & 11.5 \\
\hline 4 & & & & $\mathrm{C} 10$ & 0.067 & 10.4 & 10.2 \\
\hline 5 & & & & $\mathrm{C} 12$ & 0.078 & 8.9 & 8.5 \\
\hline 6 & & & & C14 & 0.091 & 7.6 & 7.5 \\
\hline 7 & \multirow{6}{*}{ PropylBPE } & \multirow{6}{*}{2.8} & \multirow{6}{*}{2.3} & $\begin{array}{l}\text { no am- } \\
\text { phiphile* }\end{array}$ & 0.094 & 7.4 & 6.2 \\
\hline 8 & & & & $\mathrm{C} 6$ & 0.27 & 2.5 & 2.8 \\
\hline 9 & & & & C8 & 0.33 & 2.1 & 2.5 \\
\hline 10 & & & & $\mathrm{C} 10$ & 0.35 & 2.0 & 2.3 \\
\hline 11 & & & & $\mathrm{C} 12$ & 0.40 & 1.7 & 2.1 \\
\hline 12 & & & & C14 & 0.42 & 1.6 & 2.0 \\
\hline 13 & \multirow{3}{*}{ DEGBPE } & \multirow{3}{*}{1.6} & \multirow{3}{*}{1.2} & $\begin{array}{l}\text { no am- } \\
\text { phiphile* }\end{array}$ & 0.12 & 5.7 & 5.7 \\
\hline 14 & & & & C6 & 0.035 & 20.0 & 18.0 \\
\hline 15 & & & & C14 & 0.055 & 12.7 & 11.0 \\
\hline 16 & \multirow{3}{*}{ HexylBPE } & \multirow{3}{*}{4.0} & \multirow{3}{*}{3.5} & $\begin{array}{l}\text { no am- } \\
\text { phiphile* }\end{array}$ & 0.082 & 8.5 & 7.9 \\
\hline 17 & & & & C6 & 1.9 & 0.4 & 0.7 \\
\hline 18 & & & & C14 & 3.0 & 0.2 & 0.3 \\
\hline
\end{tabular}

Table 2. $\log P$ values and kinetic parameters (reaction rate, $k$, calculated and experimental $t_{1 / 2}$ values) for the different propargyl containing substrates. $\#=$ Calculated via ChemDraw Version 18.2 ${ }^{*}=$ PBS:Acetone mixture $(1: 1 \mathrm{v} / \mathrm{v})$.

The results show a clear trend, where the more hydrophobic substrates reacted faster, indicating the significant impact of this factor on the reaction rate. The faster reaction rate can be contributed to the higher tendency of the substrates to migrate into the micellar nanoreactor as their lipophilicty increases as previously reported for other polymeric nanoreactors. $^{25,47}$ 
To estimate the relative effect of the substrate lipophilicity on the reaction rates, the calculated $k$ values for all four substrates in the presence of both C6 and C14 catalytic micelles were plotted against the substrates' logP values (Figure 5C). Importantly, when comparing the contributions of the substrate and micellar nanoreactor hydrophobicity, it appears that while the amphiphiles cLogP values showed linear correlation with the reaction rates (Figures $2 \mathrm{D}$ and 3D), the substrate's LogP values yielded exponential correlation (Figures 5C and 5D). These results imply that the substrate lipophilicity plays a more significant role in determining the rate of the reaction. Interestingly, when comparing the kinetic data for the different substrates with the C6 and C14 based nanoreactors, similar slops were observed when plotting the $\mathrm{k}$ values in logarithmic scale against the substrates' $\log \mathrm{P}$ value (Figure 5D). These results indicate that the amphiphilicity of the nanoreactors maintained its relative impact on the reaction even when the lipophilicity of the substrates changed drastically.

To ensure that the observed kinetic trends are not the result of specific metal-substrate interactions, which are independent of the hydrophobic microenvironment of the nanoreactors, we have performed a control experiment in the absent of the micelles. Instead of the micellar set-up, the reaction took place in a mixture of Acetone: PBS $(1: 1 \mathrm{v} / \mathrm{v})$ as reaction media, whereas the concentrations of the palladium acetate salt and the different substrates remained the same as for the micellar experiments. All four substrates showed relatively similar reaction rates in the range $0.08-0.12 \mathrm{~h}^{-1}$ (Table 2), which are of the same order of magnitude as the PNPPE substrate in the presence of the micellar nanoreactors (Figure 5C,5D, S30). These results demonstrate yet again the high favorability of the micellar nanoreactors towards more hydrophobic substrates, emphasizing the potential of using such systems for execution of organic reactions in aqueous environment.

A
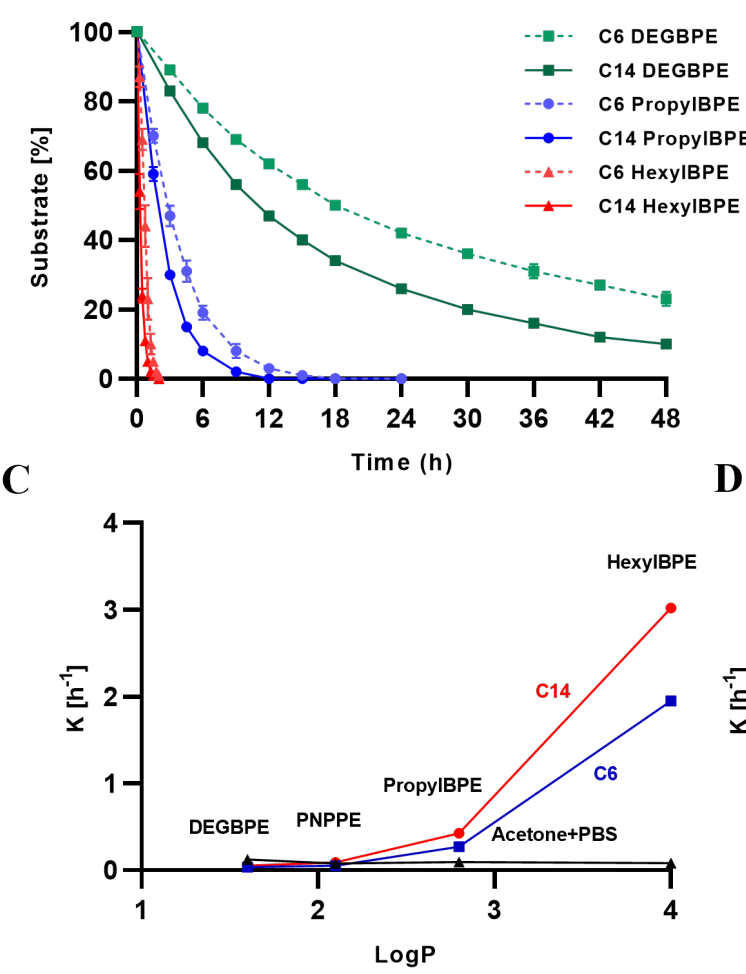

B

D
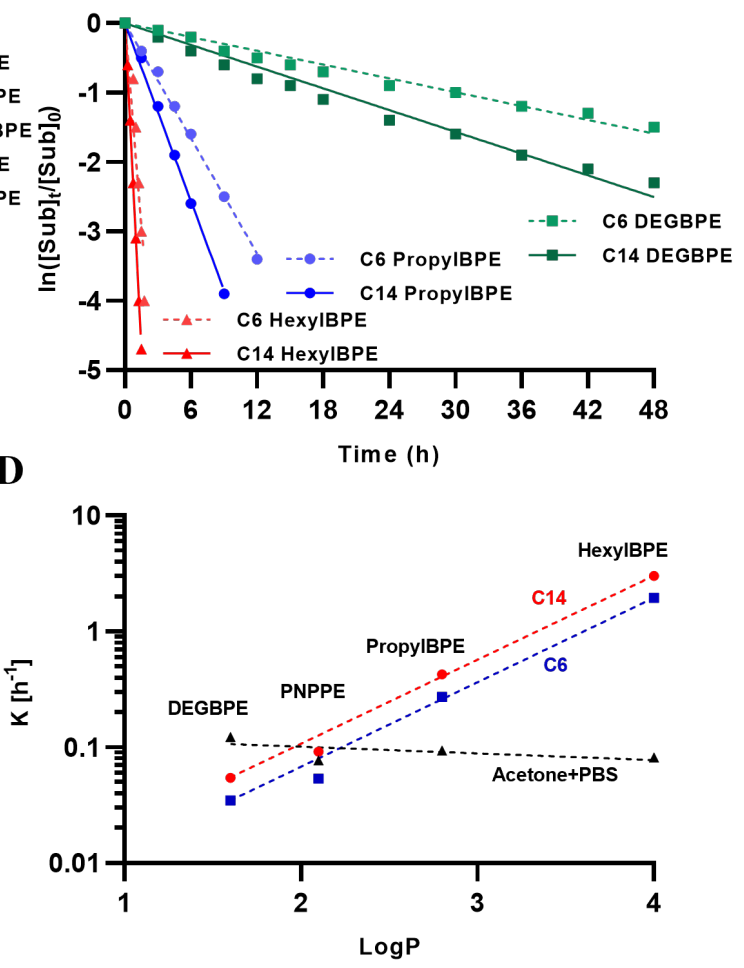

Figure 5. O-Propargyl cleavage profile of Hexyl, Propyl, PNPPE and DEG substrates; A) Normalized substrate consumption over time, in the presence of C6 (dashed lines) and C14 (full lines) metallic-micelles. [Amphiphile] $=42 \mu \mathrm{M}$; $\left[\mathrm{Pd}(\mathrm{OAc})_{2}\right]=83 \mu \mathrm{M}$; [Substrate] = $100 \mu \mathrm{M}$. B) Natural log of the normalized experimental kinetic data. C) Calculated rate constants values in the presence of C6 micelles (red), C14 micelles (blue) and Acetone+PBS (1:1 v/v) set-up (black) plotted against the substrates $\log \mathrm{P}$ values. D) Logarithmic representation of graph C. 


\title{
CONCLUSIONS
}

In this work we wished to study how the hydrophobicity of micellar nanoreactors and substrate would affect the rate of a model organo-metallic reaction being executed in water. As a model reaction we selected the bioorthogonal palladium-mediated depropargylation reaction, which has a potential for biomedical and therapeutic applications such as the activation of probe molecules and prodrugs. Our nanoreactor platform was based on palladium loaded micelles composed of PEG-dendron amphiphiles. To evaluate the influence of changes in the lipophilicity of both the nanoreactor and the substrate on the reaction rate, we synthesized a small library of five amphiphiles, which varied in the length of their aliphatic end-groups, and four propargyl-modified substrates with varying degree of hydrophobicity.

Kinetic analysis of the experimental data highlighted the crucial effect that the amphiphiles composing the nanoreactor have on the reaction rate, where the more hydrophobic hybrids yielded faster kinetics. Taking advantage of the high molecular precision, which emerges from the utilization of dendrons as the hydrophobic core forming block, we finetuned the hydrophobicity of the amphiphiles by adjusting the number of carbons in the aliphatic end-groups. This molecular precision allowed us to demonstrate how small structural changes of only few carbons, have a significant impact on the reaction rate. Interestingly, we found a linear correlation between the cLogP values of the dendritic block and the reaction rate, and observed that this trend is not limited to a specific type of substrate.

In addition, we showed that the hydrophobicity of the substrate had even a bigger impact on the kinetics of the model reaction, as exponential correlation was found between the $\log \mathrm{P}$ values of the substrates and the observed reaction rates. These results together with the kinetics for a control reaction media composed of acetone/water mixture, which showed similar rates for all substrates, indicated on the high selectivity of the micellar nanoreactors toward the more hydrophobic substrates. Understanding the mutual contributions of the hydrophobicity of both the substrate and the micellar microenvironment on the reaction rate, provides essential knowledge towards the rational design of nanoreactor systems, for fields ranging from green chemistry to therapeutic applications involving bioorthogonal catalysis for the activation of prodrugs in living systems.

\section{ASSOCIATED CONTENT}

\section{Supporting Information}

Synthetic procedures, amphiphiles and micelles characterization data, detailed experimental protocols and control experiments. The Supporting Information is available free of charge at XXX

\section{AUTHOR INFORMATION}

\section{Corresponding Author}

*amirroey@tauex.tau.ac.il

ORCID Roey J. Amir: 0000-0002-8502-3302

\section{Author Contributions}

\author{
† S.T. and S.S.W. contributed equally.
}

\section{Funding Sources}

This project has received funding from the European Union's Horizon 2020 research and innovation programme under the Marie Sklodowska-Curie grant agreement No. 765497 (THERACAT).

\section{ACKNOWLEDGMENT}

S.T. thanks the ADAMA Center for Novel Delivery Systems in Crop Protection, Tel-Aviv University for the financial support. G.S. thanks the Marian Gertner Institute for Medical Nanosystems in Tel Aviv University for their financial support.

\section{ABBREVIATIONS}

CMC, critical micelle concentration; DLS, dynamic light scattering; HPLC, high-pressure liquid chromatography. 


\section{REFERENCES}

(1) Wang, J.; Cheng, B.; Li, J.; Zhang, Z.; Hong, W.; Chen, X.; Chen, P. R. Chemical Remodeling of Cell-Surface Sialic Acids through a Palladium-Triggered Bioorthogonal Elimination Reaction. Angew. Chemie Int. Ed. 2015, 54 (18), $5364-5368$.

Szponarski, M.; Schwizer, F.; Ward, T. R.; Gademann, K. On-Cell Catalysis by Surface Engineering of Live Cells with an Artificial Metalloenzyme. Commun. Chem. 2018, 1 (1), 84.

Liu, Y.; Pujals, S.; Stals, P. J. M.; Paulöhrl, T.; Presolski, S. I.; Meijer, E. W.; Albertazzi, L.; Palmans, A. R. A. Catalytically Active Single-Chain Polymeric Nanoparticles: Exploring Their Functions in Complex Biological Media. J. Am. Chem. Soc. 2018, 140 (9), 3423-3433.

Weiss, J. T.; Dawson, J. C.; Macleod, K. G.; Rybski, W.; Fraser, C.; Torres-Sánchez, C.; Patton, E. E.; Bradley, M.; Carragher, N. O.; Unciti-Broceta, A. Extracellular Palladium-Catalysed Dealkylation of 5-Fluoro-1-Propargyl-Uracil as a Bioorthogonally Activated Prodrug Approach. Nat. Commun. 2014, 5.

Yusop, R. M.; Unciti-Broceta, A.; Johansson, E. M. V.; Sánchez-Martín, R. M.; Bradley, M. Palladium-Mediated Intracellular Chemistry. Nat. Chem. 2011, 3 (3), 239-243.

Tonga, G. Y.; Jeong, Y.; Duncan, B.; Mizuhara, T.; Mout, R.; Das, R.; Kim, S. T.; Yeh, Y. C.; Yan, B.; Hou, S.; Rotello, V. M. Supramolecular Regulation of Bioorthogonal Catalysis in Cells Using Nanoparticle-Embedded Transition Metal Catalysts. Nat. Chem. 2015, 7 (7), 597-603.

Unciti-Broceta, A.; Johansson, E. M. V.; Yusop, R. M.; Sánchez-Martín, R. M.; Bradley, M. Synthesis of Polystyrene Microspheres and Functionalization with Pd0 Nanoparticles to Perform Bioorthogonal Organometallic Chemistry in Living Cells. Nat. Protoc. 2012, 7 (6), 1207-1218.

Gallou, F.; Isley, N. A.; Ganic, A.; Onken, U.; Parmentier, M. Surfactant Technology Applied toward an Active Pharmaceutical Ingredient: More than a Simple Green Chemistry Advance. Green Chem. 2016, 18 (1), 14-19.

Tu, J.; Xu, M.; Franzini, R. M. Dissociative Bioorthogonal Reactions. ChemBioChem 2019, 20 (13), $1615-1627$.

For Organic Chemists, Micellar Chemistry Could Be a Revolution. C\&EN Glob. Enterp. 2020, 98 (22), 20-23.

Lipshutz, B. H. The Nano-to-Nano Effect Applied to Organic Synthesis in Water. Johnson Matthey Technol. Rev. 2017, 61, 196202.

Lipshutz, B. H.; Ghorai, S.; Abela, A. R.; Moser, R.; Nishikata, T.; Duplais, C.; Krasovskiy, A.; Gaston, R. D.; Gadwood, R. C. TPGS-750-M: A Second-Generation Amphiphile for Metal-Catalyzed Cross-Couplings in Water at Room Temperature. J. Org. Chem. 2011, 76 (11), 4379-4391.

Pang, H.; Hu, Y.; Yu, J.; Gallou, F.; Lipshutz, B. H. Water-Sculpting of a Heterogeneous Nanoparticle Precatalyst for MizorokiHeck Couplings under Aqueous Micellar Catalysis Conditions. J. Am. Chem. Soc. 2021, 143 (9), 3373-3382.

Cortes-Clerget, M.; Akporji, N.; Zhou, J.; Gao, F.; Guo, P.; Parmentier, M.; Gallou, F.; Berthon, J. Y.; Lipshutz, B. H. Bridging the Gap between Transition Metal- and Bio-Catalysis via Aqueous Micellar Catalysis. Nat. Commun. 2019, 10 (1), 1-10.

Takale, B. S.; Thakore, R. R.; Casotti, G.; Li, X.; Gallou, F.; Lipshutz, B. H. Mild and Robust Stille Reactions in Water Using Parts Per Million Levels of a Triphenylphosphine-Based Palladacycle. Angew. Chemie Int. Ed. 2021, 60 (8), $4158-4163$.

Lipshutz, B. H.; Aguinaldo, G. T.; Ghorai, S.; Voigtritter, K. Olefin Cross-Metathesis Reactions at Room Temperature Using the Nonionic Amphiphile "PTS”: Just Add Water. Org. Lett. 2008, 10 (7), 1325-1328.

Bu, M.; Cai, C.; Gallou, F.; Lipshutz, B. H. PQS-Enabled Visible-Light Iridium Photoredox Catalysis in Water at Room Temperature. Green Chem. 2018, 20 (6), 1233-1237.

Artar, M.; Souren, E. R. J.; Terashima, T.; Meijer, E. W.; Palmans, A. R. A. Single Chain Polymeric Nanoparticles as Selective Hydrophobic Reaction Spaces in Water. ACS Macro Lett. 2015, 4 (10), 1099-1103.

Liu, Y.; Turunen, P.; De Waal, B. F. M.; Blank, K. G.; Rowan, A. E.; Palmans, A. R. A.; Meijer, E. W. Catalytic Single-Chain Polymeric Nanoparticles at Work: From Ensemble towards Single-Particle Kinetics. Mol. Syst. Des. Eng. 2018, 3 (4), $609-618$.

Weiss, J. T.; Carragher, N. O.; Unciti-Broceta, A. Palladium-Mediated Dealkylation of N-Propargyl-Floxuridine as a Bioorthogonal Oxygen-Independent Prodrug Strategy. Sci. Rep. 2015, 5 (1), 9329.

Pérez-López, A. M.; Rubio-Ruiz, B.; Valero, T.; Contreras-Montoya, R.; Álvarez de Cienfuegos, L.; Sebastián, V.; Santamaría, J.; Unciti-Broceta, A. Bioorthogonal Uncaging of Cytotoxic Paclitaxel through Pd Nanosheet-Hydrogel Frameworks. J. Med. Chem. 2020, 63 (17), 9650-9659.

Sancho-Albero, M.; Rubio-Ruiz, B.; Pérez-López, A. M.; Sebastián, V.; Martín-Duque, P.; Arruebo, M.; Santamaría, J.; UncitiBroceta, A. Cancer-Derived Exosomes Loaded with Ultrathin Palladium Nanosheets for Targeted Bioorthogonal Catalysis. Nat. Catal. 2019, 2 (10), 864-872.

van de L'Isle, M. O. N.; Ortega-Liebana, M. C.; Unciti-Broceta, A. Transition Metal Catalysts for the Bioorthogonal Synthesis of Bioactive Agents. Curr. Opin. Chem. Biol. 2021, 61, 32-42.

Rubio-Ruiz, B.; Weiss, J. T.; Unciti-Broceta, A. Efficient Palladium-Triggered Release of Vorinostat from a Bioorthogonal Precursor. J. Med. Chem. 2016, 59 (21), 9974-9980.

Chen, J.; Wang, J.; Bai, Y.; Li, K.; Garcia, E. S.; Ferguson, A. L.; Zimmerman, S. C. Enzyme-like Click Catalysis by a CopperContaining Single-Chain Nanoparticle. 2018, 140 (42), 13695-13702.

Chen, J.; Wang, J.; Li, K.; Wang, Y.; Gruebele, M.; Ferguson, A. L.; Zimmerman, S. C. Polymeric "Clickase” Accelerates the Copper Click Reaction of Small Molecules, Proteins, and Cells. J. Am. Chem. Soc. 2019, 141 (24), 9693-9700. 
(27) Bai, Y.; Feng, X.; Xing, H.; Xu, Y.; Kim, B. K.; Baig, N.; Zhou, T.; Gewirth, A. A.; Lu, Y.; Oldfield, E.; Zimmerman, S. C. A Highly Efficient Single-Chain Metal-Organic Nanoparticle Catalyst for Alkyne-Azide “Click” Reactions in Water and in Cells. J. Am. Chem. Soc. 2016, 138 (35), 11077-11080.

(28) Ansari, T. N.; Taussat, A.; Clark, A. H.; Nachtegaal, M.; Plummer, S.; Gallou, F.; Handa, S. Insights on Bimetallic Micellar Nanocatalysis for Buchwald-Hartwig Aminations. ACS Catal. 2019, 9 (11), 10389-10397.

(29) Bihani, M.; Ansari, T. N.; Finck, L.; Bora, P. P.; Jasinski, J. B.; Pavuluri, B.; Leahy, D. K.; Handa, S. Scalable $\alpha$-Arylation of Nitriles in Aqueous Micelles Using Ultrasmall Pd Nanoparticles: Surprising Formation of Carbanions in Water. ACS Catal. 2020, 10 (12), 6816-6821.

(30) Sabatino, V.; Rebelein, J. G.; Ward, T. R. "Close-to-Release”: Spontaneous Bioorthogonal Uncaging Resulting from RingClosing Metathesis. J. Am. Chem. Soc. 2019, 141 (43), 17048-17052.

(31) Villarino, L.; Chordia, S.; Alonso-Cotchico, L.; Reddem, E.; Zhou, Z.; Thunnissen, A. M. W. H.; Maréchal, J.-D.; Roelfes, G. Cofactor Binding Dynamics Influence the Catalytic Activity and Selectivity of an Artificial Metalloenzyme. ACS Catal. 2020, 10 (20), 11783-11790.

(32) Destito, P.; Sousa-Castillo, A.; Couceiro, J. R.; López, F.; Correa-Duarte, M. A.; Mascareñas, J. L. Hollow Nanoreactors for PdCatalyzed Suzuki-Miyaura Coupling and O-Propargyl Cleavage Reactions in Bio-Relevant Aqueous Media. Chem. Sci. 2019, 10 (9), 2598-2603.

(33) Pal, M.; Parasuraman, K.; Yeleswarapu, K. R. Palladium-Catalyzed Cleavage of O/N-Propargyl Protecting Groups in Aqueous Media under a Copper-Free Condition. Org. Lett. 2003, 5 (3), 349-352.

(34) Patterson, D. M.; Nazarova, L. A.; Prescher, J. A. Finding the Right (Bioorthogonal) Chemistry. ACS Chem. Biol. 2014, 9 (3), 592-605.

(35) Ramil, C. P.; Lin, Q. Bioorthogonal Chemistry: Strategies and Recent Developments. Chem. Commun. 2013, 49 (94), 1100711022.

(36) Sletten, E. M.; Bertozzi, C. R. Bioorthogonal Chemistry: Fishing for Selectivity in a Sea of Functionality. Angew. Chemie - Int. Ed. 2009, 48 (38), 6974-6998.

(37) Bertozzi, C. R. A Decade of Bioorthogonal Chemistry. Acc. Chem. Res. 2011, 44 (9), 651-653.

(38) Devaraj, N. K. The Future of Bioorthogonal Chemistry. ACS Cent. Sci. 2018, 4 (8), 952-959.

(39) Hang, H. C.; Yu, C.; Kato, D. L.; Bertozzi, C. R. A Metabolic Labeling Approach toward Proteomic Analysis of Mucin-Type OLinked Glycosylation. Proc. Natl. Acad. Sci. U. S. A. 2003, 100 (25), 14846-14851.

(40) Li, J.; Chen, P. R. Development and Application of Bond Cleavage Reactions in Bioorthogonal Chemistry. Nat. Chem. Biol. 2016, 12 (3), 129-137.

(41) Rambabu, D.; Bhavani, S.; Swamy, N. K.; Basaveswara Rao, M. V.; Pal, M. Pd/C-Mediated Depropargylation of Propargyl Ethers/Amines in Water. Tetrahedron Lett. 2013, 54 (9), 1169-1173.

(42) Coelho, S. E.; Schneider, F. S. S.; De Oliveira, D. C.; Tripodi, G. L.; Eberlin, M. N.; Caramori, G. F.; De Souza, B.; Domingos, J. B. Mechanism of Palladium(II)-Mediated Uncaging Reactions of Propargylic Substrates. ACS Catal. 2019, 9 (5), 3792-3799.

(43) Huang, R.; Li, C. H.; Cao-Milán, R.; He, L. D.; Makabenta, J. M.; Zhang, X.; Yu, E.; Rotello, V. M. Polymer-Based Bioorthogonal Nanocatalysts for the Treatment of Bacterial Biofilms. J. Am. Chem. Soc. 2020, 142 (24), 10723-10729.

(44) Gillies, E. R.; Jonsson, T. B.; Fréchet, J. M. J. Stimuli-Responsive Supramolecular Assemblies of Linear-Dendritic Copolymers. J. Am. Chem. Soc. 2004, 126 (38), 11936-11943.

(45) Harnoy, A. J.; Buzhor, M.; Tirosh, E.; Shaharabani, R.; Beck, R.; Amir, R. J. Modular Synthetic Approach for Adjusting the Disassembly Rates of Enzyme-Responsive Polymeric Micelles. Biomacromolecules 2017, 18 (4), 1218-1228.

(46) Segal, M.; Avinery, R.; Buzhor, M.; Shaharabani, R.; Harnoy, A. J.; Tirosh, E.; Beck, R.; Amir, R. J. Molecular Precision and Enzymatic Degradation: From Readily to Undegradable Polymeric Micelles by Minor Structural Changes. J. Am. Chem. Soc. 2017, 139 (2), 803-810.

(47) Chen, J.; Wang, J.; Li, K.; Wang, Y.; Gruebele, M.; Ferguson, A. L.; Zimmerman, S. C. Polymeric “Clickase” Accelerates the Copper Click Reaction of Small Molecules, Proteins, and Cells. J. Am. Chem. Soc. 2019, 141 (24), 9693-9700. 